\title{
RECENT TRENDS IN COMPUTER SCIENCE AND ENGINEERING (RTCSE)
}

\section{Jason Levy}

University of Hawaii, (USA).

E-mail: jlevy@hawaii.edu ORCID: https://orcid.org/0000-0002-9978-5412

Bishwajeet Pandey

Gyancity Research Consultancy, (India).

E-mail: gyancity@gyancity.com ORCID: https://orcid.org/0000-0001-5593-8985

Bhawani Shankar Chowdhry

Mehran University of Engineering and Technology (MUET), (Pakistan). E-mail: bhawani.chowdhry@faculty.muet.edu.pk ORCID: https://orcid.org/0000-0002-4340-9602

Giro Rodriguez

Universidad Nacional Mayor de San Marcos, (Perú). E-mail: crodriguezro@unmsm.edu.pe ORCID: https://orcid.org/0000-0003-2112-1349

\section{Gitación sugerida Suggested citation}

Levy, J., Pandey, B., Chowdhry, B. S., y Rodriguez, C. (2020). Prologue: Recent trends in computer science and engineering (RTCSE). 3C Tecnología. Glosas de innovación aplicadas a la pyme. Edición Especial, Abril 2020, 19-25. http:// doi.org/10.17993/3ctecno.2020.specialissue5.19-25 
This set of distinguished seventeen papers strengthens interdisciplinary linkages between the fields of engineering decision making, emerging computer science technologies and pandemic planning. Rather than separating the engineering and computer science technologies from their 'real world' impact, those contributing to this special issue have demonstrated attention to the engineering decision making issues that their work informs. Accordingly, this special issue helps engineering and computer science academics and practitioners to better understand the complex relationships related to intertwined and complex topics ranging from engineering reliability, environmental risk and health crises. In summary, this distinguished, cross-disciplinary, valuable, timely and international collection of seventeen papers examines the most challenging societal-technologic dilemmas facing society in light of the recent black swan events facing society. The papers have a special emphasis on crafting comprehensive, sustainable and intelligent solutions to effectively transform technologic challenges with the power of Recent Trends in Computer Science and Engineering (RTCSE). It should be noted that these papers constitute a sample of the leading papers published in recent Gyancity conferences.

In the first paper Ezzy et al. (2020) discuss a solution to the problem of warehouse automation for transferring goods. To meet the demands of large scale material movement in warehouses, an automated system is proposed in lieu of a manual workforce to automate the warehouse picking process using an Automated Guided Vehicle (AGV). The system is designed such that the robot can operate on a known 'map' laid out on the warehouse floor. The system proposed is a prototype of a large-scale system and demonstrates proof of concept of the overall AGV system for warehouse management. The system performance is measured in a straight-line movement and once the robot turns at specific degrees. A metal track was built via metal strips and was then detected by the proximity sensors. This process is also very robust and can withstand the demands of an industrial setting.

In the second paper, Urooj et al. (2020) provides a discussion of water crises: they note that one large amount of residential and industrial are wasted every year. They created a Kivy application for the user in which they can control the fluid waste problem with the integration of hardware that includes Microcontrollers, Ultrasonic sensors, Relay shields, node MCU 8266, and a contactor. With their designed application, users can control the water level status, motor accessibility (on/off), the status of water consumption, and 
message alerts. In future work, they seek to enhance the water model and apply it to the agriculture sectors.

In the third paper, Talha et al. (2020) put forward a unique turbine that provides optimal electrical output. The authors note that a water turbine is one of the key technologies used in producing green energy which benefits both the environment and society. The design of the turbine is such that when water encounters the blade, the turbine begins to rotate to generate electricity. It is proposed that the model can be improved if the joint resistance is addressed by adding a ball bearing that will increase the motion. Future designs of the turbine can be improved by fully submerging the turbine instead of partial submersion without the detriment of ease in installation and maintenance. The design can further be implemented on other water systems such as rivers and canals by changing the size of the turbine.

In the fourth paper, Tiwari, Kumar and Sharan (2020) examines the concept of the reversible logic: it depends on the dominant modules of a processor. The authors note that the shift register can be studied with the use of the reversible logic which can shift the bits of the information towards both side i.e. left and right. In order to analyze the quantum processor, the Quantum Computational Automata (QCA) as well as Verilog software is used to simulate the parameters of the device and then obtain the characteristics of the device. The authors take a reversible logical computing based approach that can be used in the ALU of a quantum processor with the help of D-type FFs. This circuit is designed and analyzed for key parameters such as the size of the cell, the number of cells used, delay, temperature dependency, power dissipation, etc. The proposed circuit acts as an alternative to the CMOS technology, and is analyzed for a typical range of power dissipation (650$750 \mathrm{meV})$ and temperature $\left(1^{\circ} \mathrm{K}-10^{\circ} \mathrm{K}\right)$.

In the fifth paper, Memon et al. (2020 provide an overview of buck-boost converter technologies.

The authors note that the buck-boost converter operating in critical conduction mode (CRM) is commonly utilized in various applications. The system has a number of advantages: protection against short circuiting, minimum component count, low operating duct-cycles, and low voltage, MOSFETs. Etc. A new control scheme of variable on-time 
control (VOTC) is proposed in this paper. The VOTC can be implemented by modulating the turn-on time of the buck-boost switch.

Simulation results are presented to verify the effectiveness of the proposed control strategy. In the sixth paper, Shah and Abosaq (2020) discuss a novel approach: the concept of powering systems wirelessly. The authors note that in the 19th century, Nikola developed 'Tesla Tower' in hope of transferring power wirelessly. The authors discuss WPT using Inductive Coupling which falls under the domain of NFWPT: a transmitter coil is used to transmit power to the receiver coil via a magnetic field. Inductive coupling is an efficient way to transmit power through short distances and make its way in smartphones and the health industry. This paper discusses the theoretical foundation of Inductive coupling and presents results of an experimental work done on WPT via Inductive Coupling.

In the seventh paper Hertzog and Swart (2020) propose a revolutionary technology for fishermen. The purpose of this paper is to present a cost-effective and innovative test jig that may be used to determine the reliability and consistency of operation of various fishing-bait release mechanisms. The main components of the system are a HX711 instrumentation amplifier, a load cell and an Arduino Mega microcontroller. The accuracy of the system was determined to be $99.879 \%$. Reliability values for a Gannet Sport mechanism with a $0.55 \mathrm{~mm}$ Kingfisher line ranged from $599 \mathrm{~g}$ to $642 \mathrm{~g}$, giving a maximum deviation of $43 \mathrm{~g}$.

The results provide evidence that the system is both reliable and valid.

In the eighth paper, Lehloka, Swart and Hertzog (2020) discuss issues of energy supply. The purpose of this paper is to empirically validate the optimum tilt for PV modules in the Highveld of South Africa. Three fixed-axis PV modules installed at optimum tilt angles of Latitude minus $10^{\circ}$, Latitude, and Latitude plus $10^{\circ}$ serve as the basis of this study. These optimum tilt angles are utilized based on the recommendations by Heywood and Chinnery. A key recommendation is that $\mathrm{PV}$ modules should be mounted at Latitude minus $10^{\circ}$ for the summertime period in the Highveld region of South Africa. Energy supply is a major problem in today's world due to an increase in demand, fossil fuel challenges and increase in global warming due to carbon emissions. It is shown that the efficiency of Photovoltaic (PV) 
systems is affected not only by varying environmental conditions but also by the installation of its PV modules.

In ninth, Ali et al. (2020) examine the effectiveness of tumor diagnoses. This paper facilitates radiologists in diagnosing lung tumors and helps differentiate between the types of tumor. Computed Tomography (CT)-scan images in Digital Imaging and Communications in Medicine (DICOM) format are used to identify the lung tumor (benign or malignant) using a learning algorithm. The proposed diagnostic software provides accurate results with bright CT scans: different orientations can help to increase the efficiency and accuracy of the diagnostic procedure. The proposed computer aided diagnosis can help the radiologists to detect tumors at an early stage, decrease the false positive rate, and reduce the overall cost of the diagnostic procedure.

The tenth paper involves the design of an automated pizza cutting machine in the food industry. The objective of this paper authored by Saeed, Sattar and Ferguson (2020) involves the design of a machine that would cut a pizza into even slices by a single press. The need of the product has been determined by surveys that were collected from various pizza vendors. This machine is designed to cut a pizza of any size in less than 30 seconds; increasing the overall productivity. The pneumatic system and round cutting tools are used to get the pizza cuts into even slices with better precision and more accurate shape. The stress and motion analysis conducted as part of the design procedure and their results are also discussed.

The eleventh paper, authored by Steenekamp and Swart (2020), discuss premature bearing failures due to Electrical Current Discharge (ECD). The purpose of this paper is to present an innovative jig that may be used to expose mechanical bearings to ECD, in order to clarify its associated effects on the bearing that need to be understood before any mitigating techniques can be proposed. An experimental design is used in this study. A method is presented using an ignition coil wiring harness of a vehicle to safely induce ECD across a specific bearing. Three samples were used and analyzed with an optical and electron scanning microscope. The used ball bearing exposed to ECD showed micro-cratering, a result of electric current passage. A few deep scratches and indentations were observed on the raceway surface. This is due to abrasive wear particles embedded in the raceway surface 
sliding between the major bearing components. A recommendation is made to use this innovative jig to test the impact of ECD on bearings from other suppliers.

Twelfth, Ujjan et al. (2020) note that robust and accurate adhesion level identification is crucial for proper operation of railway vehicles. In this research a solid axle Wheel-set was modeled along with different adhesion conditions and a dataset was prepared for the training of Deep Neural Networks (DNNs) in Python. Furthermore, it explored the potential of DNNs and various data driven algorithms on our noisy sequential dataset for classification tasks and achieved $91 \%$ accuracy in identification of adhesion condition with the final model.

In the thirteenth paper, in this special issue, Huapaya, Rodriguez and Esenarro (2020) describes the most prominent algorithms of Supervised Machine Learning (SML), their characteristics, and comparatives in the way of treating data. The Heart Disease dataset obtained from Kaggle was used to determine and test its highest percentage of accuracy. To achieve the objective, Python sklearn libraries were used to implement the selected algorithms, evaluate and determine which algorithm is the one that obtains the best results, applying decision tree algorithms achieved the best prediction results.

In the fourteenth paper, the effect of the COVID-19 epidemic on research activities in Engineering universities in Pakistan, are discussed by Massan, Dahri and Shaikh (2020). Their solutions via technology are also emphasized. It is shown that invasive quarantines and adept social distancing are required to mitigate the effects of the Coronavirus COVID-19 pandemic. The goal of this paper is to ensure good practices, effective command, and clear and lucid communication in order to defeat the pandemic and prevent the rise of future disasters

In the fifteenth paper, the Usability of eGovernance for the citizens of Pakistan is discussed by Dahri, Massan and Maitlo (2020). The study findings show that most of the users had good perceived usability satisfaction and only one-third of the users were rated as possessing little or poor usability. The objectivity of the results indicates the need to bridge the mismatch between the developer's perception and the user experience. 
In the sixteen paper, an extended Kalman filter is discussed. Mal et al. (2020) use this model to estimate contact forces at the wheel-rail interface. The wheel-track interface is the most significant part in the railway dynamics because the forces produced at wheel-track interface governs the dynamic behavior of entire vehicle. This contact force is complex and highly non-linear function of creep and affected with other railway vehicle parameters. The real knowledge of creep force is necessary for reliable and safe railway vehicle operation. This paper proposed model-based estimation technique to estimate non-linear wheelset dynamics. In this paper, non-linear railway wheelset is modeled and estimated using Extended Kalman Filter (EKF). Both wheelset model and EKF are developed and simulated in Simulink/MATLAB.

In the seventeenth paper, Ivanov et al. (2020) note that digital technologies are transforming the field of medical training, simulation and modeling. Advances in the field of virtual Augmented Reality (AR) and virtual simulation are described in detail, particularly as they relate to medical education and training. An overview of key medical simulation tools is provided in order provide foundational knowledge about this rapidly growing field. A timely and valuable original Augmented Realty system is put forward. 